\title{
La educación nutricional y su relación con los hábitos alimentarios en estudiantes de Ciencias de la Salud de una universidad privada del Perú
}

\author{
William Victor Cupe Cabezas ${ }^{1}$, Luis Antonio Remuzgo Barco ${ }^{2}$, Pedro Constante Costilla Castillo \\ Raúl Elías Dueñas Luján ${ }^{1,3}$ \\ 1 Universidad César Vallejo, Alfredo Mendiola 6232, Los Olivos, Lima, Perú \\ 2 Universidad Autónoma del Perú, Panamericana Sur Km 16.3, Villa El Salvador, Lima, Perú \\ ${ }^{3}$ Universidad del Pacífico, Gral. Luis Sánchez Cerro 2145, Jesús María, Lima, Perú
}

Recibido el 9 de marzo del 2020. Revisado el 3 de octubre del 2020. Aceptado el 5 de octubre del 2020.

DOI: https://doi.org/10.33017/RevECIPeru2020.0009/

\section{Resumen}

El estudio elaborado tiene como objetivo mostrar la relación existente entre la educación nutricional y los hábitos alimentarios en los estudiantes de Ciencias de la Salud de una universidad privada peruana. El enfoque de investigación es cuantitativo y el diseño de investigación es no experimental de tipo transversal. Los datos de este estudio surgen de una muestra no probabilística conformada por 342 estudiantes de la Universidad Privada del Norte de Lima, Perú que pertenecen a las carreras profesionales de Nutrición y Dietética $(n=90)$, Enfermería $(n=85)$, Obstetricia $(n=85)$ y Terapia física y rehabilitación $(n=82)$ La unidad de análisis estuvo conformada por estudiantes que cursaron la asignatura de Introducción a la Nutrición en el ciclo académico 2019-II. Se observa que del total el $47 \%$ de estudiantes de Ciencias de la Salud tienen un nivel moderado de educación nutricional, le sigue un $35 \%$ de estudiantes con un nivel bajo y un $18 \%$ posee un nivel alto de educación nutricional. Sobre los hábitos alimentarios el $39 \%$ del total de estudiantes posee un nivel moderado, seguido de un $32 \%$ que tiene un nivel bajo y un $29 \%$ posee un nivel alto. Se evidencia que los estudiantes representativos de la población poseen una educación adecuada respecto a la importancia del consumo de agua y verificación de la fecha de vencimiento de productos envasados. Además, poseen hábitos adecuados respecto al consumo de la primera comida del día y las frutas. Asimismo, se concluye que la educación nutricional recibida hasta ahora y sus hábitos alimentarios, mayoritariamente son aceptables, pero no sobresalientes. Ello implica que el conocimiento que se posee de nutrición no necesariamente influye de manera determinante en sus prácticas alimentarias. No se encontró una gran diferencia entre las carreras mencionadas respecto a la educación nutricional recibida y los hábitos alimentarios.

Descriptores: Educación nutricional, Hábitos alimentarios, Conducta alimentaria, Alimentación saludable.

\begin{abstract}
The study carried out aims to show the relationship between nutritional education and eating habits in Health Sciences students at a private Peruvian university. The research approach is quantitative and the research design is non-experimental of a cross-sectional type. The data of this study arise from a non-probabilistic sample made up of 342 students from the Universidad Privada del Norte from Lima, Peru who belong to the professional careers of Nutrition and Dietetics $(n=90)$, Nursing $(n=85)$, Obstetrics $(n=85)$ and Physical Therapy and Rehabilitation $(n=82)$ The unit of analysis was made up of students who took the subject of Introduction to Nutrition in the academic year 2019-II. It is observed that of the total, $47 \%$ of Health Sciences students have a moderate level of nutritional education, followed by $35 \%$ of students with a low level and $18 \%$ has a high level of nutritional education. Regarding eating habits, 39\% of all students have a moderate level, followed by $32 \%$ who has a low level and $29 \%$ has a high level. It is evidenced that the representative students of the population have an adequate education regarding the importance of water consumption and verification of the expiration
\end{abstract}


date of packaged products. In addition, they have adequate habits regarding the consumption of the first meal of the day and fruit. Likewise, it is concluded that the nutritional education received so far and their eating habits are mostly acceptable, but not outstanding. This implies that their knowledge of nutrition does not necessarily have a decisive influence on their eating practices. No great difference was found between the aforementioned careers regarding nutritional education received and eating habits.

\section{Keywords: Nutrition education, Food habits, Food behaviour, Healthy eating.}

\section{Introducción}

La Educación Nutricional otorga la orientación adecuada para que todo ser humano que comparte un núcleo familiar pueda alimentarse de forma tal que no perjudique su salud, más bien la beneficie. Así como también le da la posibilidad de identificar equivocadas elecciones de alimentación, prevenirlas y orientar a cualquier persona sobre las bondades de una saludable forma de alimentarse [1].

El nivel de educación nutricional de las personas influye directamente para la elección o no de algún producto alimenticio y su consumo. Un hábito alimentario adecuado puede repercutir en una excelente forma de vida y buen estado de salud [2].

Diversos estudios de investigación realizados a nivel nacional e internacional a estudiantes universitarios evidencian que no tienen buenos hábitos de alimentación. Los estudiantes universitarios de la Universidad pública San Luis Gonzaga de Ica poseen formas inadecuadas de alimentarse, puesto que ingieren productos que poseen un porcentaje alto de calorías. Esto unido a la ausencia de actividad física repercute en su salud [3].

Estudiantes de una universidad del ámbito privado de Lima consumen comida denominada "rápida" y bebidas gasificadas de manera frecuente: tres a cuatro veces por semana en promedio [4].

La comunidad universitaria identifica su forma de alimentación y estilo de vida con un inadecuado régimen de comidas, ya que prevalece el consumo de bebidas gasificadas y comida alta en calorías que repercute para que no se produzca una alimentación equilibrada necesaria para la realización de actividades que contribuyan a su desarrollo profesional [5].

Toda costumbre adquirida por vía familiar en la alimentación es una herencia que suele copiarse de generación en generación. Aunque debido al avance de diversos aspectos sociales como la tecnología y la presión por conseguir metas a corto y mediano plazo influye en la elección de formas de alimentarse que pueden producir un impacto enorme en la salud de los estudiantes universitarios [6].

Si es mayor la formación nutricional de una persona, entonces tendrá un mejor hábito alimentario, sin embargo, ciertos factores como los culturales o económicos producen un nuevo patrón alimentario [7].

La forma de alimentación de una persona puede influir en su salud de manera directa, así como también es una manera de lograr prevenir enfermedades degenerativas. Actualmente, se conoce que los nutrientes presentes en los alimentos adecuados influyen en el mantenimiento de la salud. [8].

Una alimentación adecuada y el ejercicio son fundamentales para aumentar paulatinamente la salud a nivel físico y mental de las personas, favorecer la reducción de los niveles de estrés para que estas se sientan mejor y disminuye significativamente la mortalidad. Si no se tiene una alimentación idónea, ello puede repercutir negativamente en la salud. En países europeos se observa que una práctica alimentaria inadecuada puede afectar a los adolescentes y producir consecuencias negativas a largo plazo [9].

Los hábitos alimentarios son adquiridos por la influencia del medio social y familiar. Así como también precisar que las instituciones educativas son un escenario ideal para reforzar por medio del docente prácticas beneficiosas de alimentación para el estudiante [10].

Las acciones de educación nutricional desarrolladas en el medio familiar contribuyen a la formación de patrones saludables y son buen patrimonio cultural de partida sobre el que se asienta las intervenciones educativas posteriores [11].

Instituciones en nuestro país, como el Ministerio de Salud organiza campañas constantes que buscan modificar conductas erróneas que realizan personas en su alimentación, así como también 
reglamentos y leyes que den a conocer advertencias en el etiquetado de los productos para que las personas puedan conocer el contenido del mismo.

La educación nutricional se aprende en el hogar, aunque a veces no es la adecuada por la ignorancia involuntaria expresada al preparar los alimentos. En diversas universidades muchas carreras están incluidas en la denominación "Ciencias de la Salud" en la que se les orienta a los estudiantes de manera conceptual y práctica para que obtengan capacidades cuyo objetivo es el cuidado y bienestar de las personas. Por lo tanto, dichos estudiantes deben recibir no solo una adecuada formación, sino ser conscientes que el ser instruido en la correcta alimentación es un pilar fundamental en el cuidado humano.

Considerando lo expresado anteriormente, la presente labor investigativa tiene como finalidad general determinar la relación que puede existir entre la educación nutricional y los hábitos alimentarios de los estudiantes universitarios de Ciencias de la Salud de una universidad privada de Lima.

\section{Metodología}

Esta investigación hizo uso del enfoque cuantitativo debido a que los planteamientos a investigar son delimitados y además específicos Asimismo, el alcance de la investigación fue correlacional. Se recurrió al diseño de investigación no experimental de tipo transversal. [12].

El criterio de selección fue probabilístico ya que fueron necesario dos procedimientos. El primero: desarrollar el cálculo del tamaño muestral con un nivel de confianza del $95 \%$ y un margen de error del $5 \%$ para que sea representativo a la población de 3144 estudiantes de Ciencias de la Salud. El segundo: seleccionar los elementos muestrales de tal forma que todos tengan la posibilidad de ser elegidos.

\subsection{Sujetos}

La muestra para el presente estudio realizado antes de la finalización de la asignatura Introducción a la Nutrición estuvo conformada por 342 estudiantes de la Universidad Privada del Norte (Lima) que pertenecen a las carreras profesionales de Nutrición y Dietética $(n=90)$, Enfermería $(n=85)$, Obstetricia $(\mathrm{n}=85)$ y Terapia física y rehabilitación $(\mathrm{N}=82) \mathrm{La}$ unidad de análisis estuvo conformada por estudiantes que cursaron la asignatura en mención en un ciclo académico, específicamente el 2019 II.

\subsection{Técnica}

La técnica empleada para recabar los datos fue la encuesta. Dicha técnica permitió la recolección de datos sobre las variables Educación nutricional y Hábitos alimentarios de los estudiantes de Ciencias de la Salud de la universidad mencionada. El instrumento que se utilizó para ello fue el cuestionario porque a través de un conjunto de preguntas cerradas se recolectó datos con respecto a las variables a medir.

\subsection{Instrumento}

El instrumento utilizado tuvo que ser evaluado por un juicio de expertos para determinar su validez. Los docentes que participaron en la realización de la evaluación fueron de Ciencias de la Salud por sus conocimientos en investigación para que -de esa forma- puedan juzgar de manera imparcial la idoneidad de las preguntas del instrumento, en base a la congruencia de contenido y la claridad al ser redactada. La encuesta fue adaptada en base al cuestionario de Torres, Trujillo, Urquiza, Salazar y Taype. Se eligió las principales preguntas de acuerdo a los objetivos planteados. En el reporte estadístico Alpha de Cronbach, se obtuvo un índice de ,802 lo que expresa que el instrumento presenta una alta confiabilidad.

\subsection{Proceso de recolección de datos}

La recolección de datos se realizó a la muestra mencionada en un solo momento, previamente se tramitó el permiso para ser presentado a las autoridades de la Universidad para aplicar el cuestionario que se realizó en el mes de setiembre a inicio de cada actividad académica y coordinado con los diversos docentes de Ciencias de la Salud de la carreras profesionales mencionadas para-de esta forma- no interrumpir sus funciones. Se les explicó a los estudiantes los motivos e importancia de la investigación y la forma del llenado del cuestionario antes de su desarrollo cuyas respuestas fueron marcadas en una escala tipo Likert, en donde (1) indicaba Nunca, (2) Casi nunca, (3) A veces, (4) Casi siempre y (5) Siempre. Luego de la explicación de las instrucciones e indicaciones pertinentes se les dio el tiempo necesario para responder a los reactivos que en promedio les tomó 15 a 20 minutos. 
La presente investigación tuvo un enfoque basado en la cuantificación de los resultados obtenidos a través del cuestionario, por ello el método correspondiente para el análisis de los datos es el cuantitativo. El procesamiento de los resultados que se obtuvieron de las respuestas que emitieron los sujetos de la muestra tuvo un procesamiento estadístico basado en la aplicación del software Excel 2010, seguidamente la base de datos obtenida fue exportada al software estadístico SPSS v.25 donde se realizó el procesado de la información, una estructuración de niveles alto, medio bajo de educación nutricional y hábitos alimentarios por baremación y la interpretación de los resultados por medio del uso de tablas.

\section{Resultados}

Se observa en la Tabla 1 que del total, el $47 \%$ de estudiantes de Ciencias de la Salud tienen un nivel moderado de educación nutricional, le sigue un 35\% de estudiantes con nivel bajo y un $18 \%$ posee un nivel alto de educación nutricional.

Tabla 1: Niveles de Educación Nutricional de los estudiantes de Ciencias de la Salud

\begin{tabular}{|c|c|c|c|c|c|c|c|}
\hline & & & \multicolumn{4}{|c|}{ Escuela Académica Profesional } & \multirow[b]{2}{*}{ Total \% } \\
\hline & & & Nutrición & Enfermería & Obstetricia & Terapia física & \\
\hline \multirow{6}{*}{ Niveles } & \multirow[b]{2}{*}{ Bajo } & $\mathrm{N}^{\circ}$ & 16 & 28 & 39 & 36 & 119 \\
\hline & & $\%$ & $5 \%$ & $8 \%$ & $11 \%$ & $11 \%$ & $35 \%$ \\
\hline & \multirow[b]{2}{*}{ Moderado } & $\mathrm{N}^{\circ}$ & 37 & 49 & 37 & 37 & 160 \\
\hline & & $\%$ & $11 \%$ & $14 \%$ & $11 \%$ & $11 \%$ & $47 \%$ \\
\hline & \multirow[b]{2}{*}{ Alto } & $\mathrm{N}^{\circ}$ & 37 & 8 & 9 & 9 & 63 \\
\hline & & $\%$ & $11 \%$ & $2 \%$ & $3 \%$ & $3 \%$ & $18 \%$ \\
\hline \multirow{2}{*}{ Total } & & $\mathrm{N}^{\circ}$ & 90 & 85 & 85 & 82 & 342 \\
\hline & & $\%$ & $26 \%$ & $25 \%$ & $25 \%$ & $24 \%$ & $100 \%$ \\
\hline
\end{tabular}

Sobre la variable hábitos alimentarios, por lo que se expresa en la Tabla 2 , el $39 \%$ del total de estudiantes posee un nivel moderado, seguido de un $32 \%$ que tiene un nivel bajo y un $29 \%$ posee un nivel alto de hábitos alimentarios.

Tabla 2 :Niveles de Hábitos Alimentarios de los Estudiantes de Ciencias de la Salud

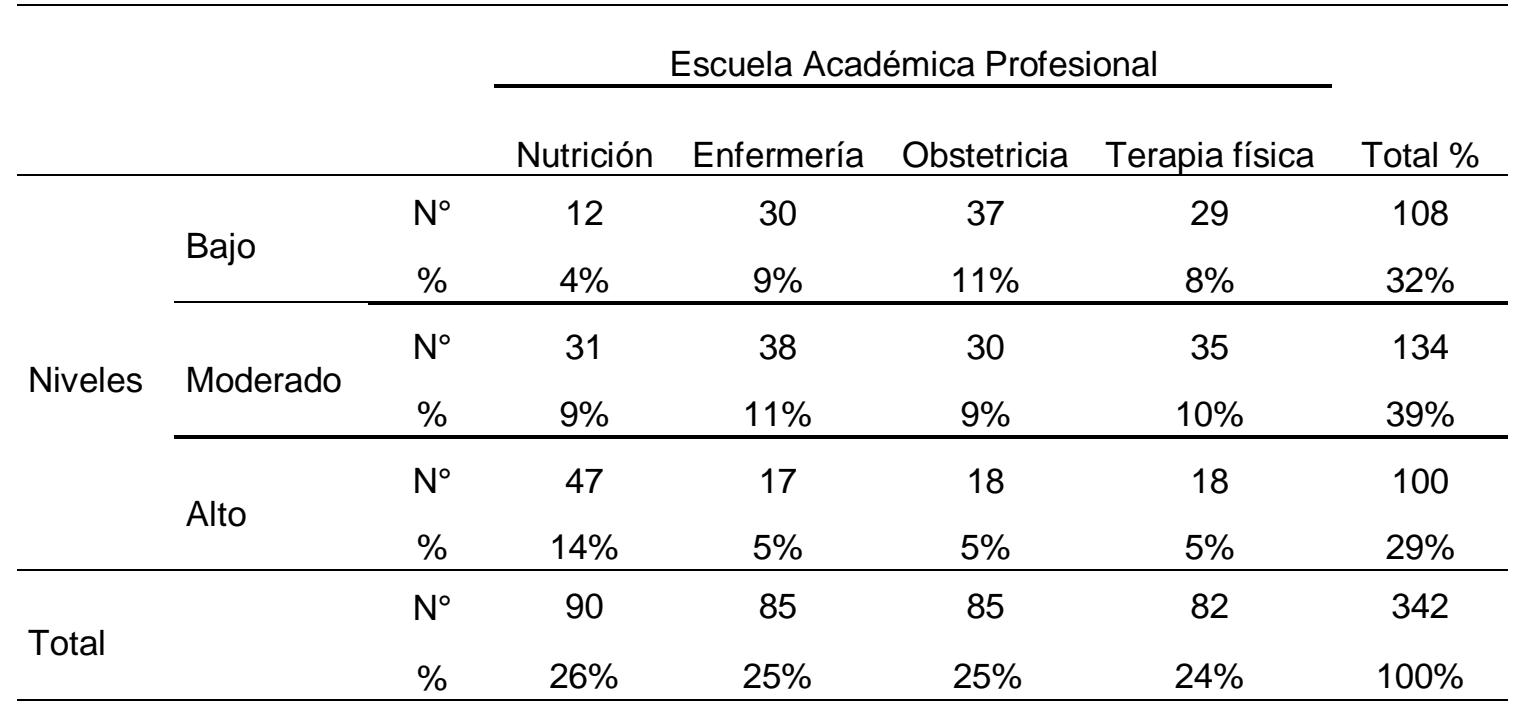


Sobre la educación nutricional, y considerando las interrogantes realizadas, el $35 \%$ del total de estudiantes encuestados de Ciencias de la Salud expresan que a veces toman ocho vasos de agua que equivale a casi dos litros. El $46 \%$ suele consumir productos procesados como dulces, galletas o chocolates también a veces. El 66\% siempre toma en cuenta la fecha de vencimiento antes de consumir un producto envasado.

Sobre los hábitos alimentarios y teniendo en cuenta las preguntas realizadas, el $43 \%$ de estudiantes de Ciencias de la Salud siempre desayuna, el 33\% casi siempre consume frutas a diario. Solo el $11 \%$ de estudiantes encuestados de Ciencias de la Salud tiene cinco momentos de comida al día. Dichos resultados se encuentran detallados en la Tabla 3.

\section{Interpretación y análisis}

Tabla 3: Análisis de los resultados obtenidos de la encuesta realizada a estudiantes de Ciencias de la Salud

\begin{tabular}{|c|c|c|c|c|c|c|c|c|}
\hline Educación nutricional & EAP & & Nunca & Casi nunca & A veces & Casi siempre & Siempre & Total \% \\
\hline \multirow{8}{*}{$\begin{array}{l}\text { Consumo de ocho } \\
\text { vasos de agua }\end{array}$} & \multirow{2}{*}{ Nutrición } & $\mathrm{N}^{\circ}$ & 1 & 11 & 31 & 25 & 22 & 90 \\
\hline & & $\%$ & $1 \%$ & $12 \%$ & $34 \%$ & $28 \%$ & $24 \%$ & $100 \%$ \\
\hline & \multirow{2}{*}{ Enfermería } & $\mathrm{N}^{\circ}$ & 2 & 20 & 33 & 17 & 13 & 85 \\
\hline & & $\%$ & $2 \%$ & $24 \%$ & $39 \%$ & $20 \%$ & $15 \%$ & $100 \%$ \\
\hline & \multirow{2}{*}{ Obstetricia } & $\mathrm{N}^{\circ}$ & 2 & 32 & 32 & 14 & 5 & 85 \\
\hline & & $\%$ & $2 \%$ & $38 \%$ & $38 \%$ & $16 \%$ & $6 \%$ & $100 \%$ \\
\hline & \multirow{2}{*}{$\begin{array}{l}\text { Terapia } \\
\text { física }\end{array}$} & $\mathrm{N}^{\circ}$ & 4 & 28 & 24 & 15 & 11 & 82 \\
\hline & & $\%$ & $5 \%$ & $34 \%$ & $29 \%$ & $18 \%$ & $13 \%$ & $100 \%$ \\
\hline \multirow[t]{2}{*}{ Total } & & $\mathrm{N}^{\circ}$ & 9 & 91 & 120 & 71 & 51 & 342 \\
\hline & & $\%$ & $3 \%$ & $27 \%$ & $35 \%$ & $21 \%$ & $15 \%$ & $100 \%$ \\
\hline \multirow{8}{*}{$\begin{array}{l}\text { Consumo de } \\
\text { productos } \\
\text { procesados }\end{array}$} & \multirow{2}{*}{ Nutrición } & $\mathrm{N}^{\circ}$ & 2 & 35 & 42 & 7 & 4 & 90 \\
\hline & & $\%$ & $2 \%$ & $39 \%$ & $47 \%$ & $8 \%$ & $4 \%$ & $100 \%$ \\
\hline & \multirow{2}{*}{ Enfermería } & $\mathrm{N}^{\circ}$ & 1 & 17 & 36 & 29 & 2 & 85 \\
\hline & & $\%$ & $1 \%$ & $20 \%$ & $42 \%$ & $34 \%$ & $2 \%$ & $100 \%$ \\
\hline & \multirow{2}{*}{ Obstetricia } & $\mathrm{N}^{\circ}$ & 1 & 12 & 38 & 29 & 5 & 85 \\
\hline & & $\%$ & $1 \%$ & $14 \%$ & $45 \%$ & $34 \%$ & $6 \%$ & $100 \%$ \\
\hline & \multirow{2}{*}{$\begin{array}{l}\text { Terapia } \\
\text { física }\end{array}$} & $\mathrm{N}^{\circ}$ & 2 & 17 & 40 & 21 & 2 & 82 \\
\hline & & $\%$ & $2 \%$ & $21 \%$ & $49 \%$ & $26 \%$ & $2 \%$ & $100 \%$ \\
\hline \multirow[t]{2}{*}{ Total } & & $\mathrm{N}^{\circ}$ & 6 & 81 & 156 & 86 & 13 & 342 \\
\hline & & $\%$ & $2 \%$ & $24 \%$ & $46 \%$ & $25 \%$ & $4 \%$ & $100 \%$ \\
\hline
\end{tabular}

Se realizó el análisis de los resultados más resaltantes por pregunta de cada una de las variables expresadas presentes en la Tabla 3. El agua es vida y el tener sed es un indicador cuando el cuerpo inicia un proceso involuntario de deshidratación. Por ello, diversos autores afirman que es necesario hidratarse correctamente y así mantener un equilibrio hídrico [13]. El 35\% del total de encuestados expresan que a veces toman ocho vasos de agua por día que equivale a casi dos litros, siendo los estudiantes de Enfermería, Obstetricia y Nutrición que poseen valores porcentuales altos por carrera. Evidentemente, es un hábito que siempre debe primar, para ello se debe tener la información pertinente sobre el mismo, es decir, recibir la educación debida no solo para estudiantes de Ciencias de la Salud, sino para el público en general [14]. 


\begin{tabular}{|c|c|c|c|c|c|c|c|c|}
\hline \multirow{6}{*}{$\begin{array}{l}\text { Consideración de la } \\
\text { fecha de vencimiento } \\
\text { antes de adquirir un } \\
\text { producto envasado }\end{array}$} & \multirow{2}{*}{ Nutrición } & $\mathrm{N}^{\circ}$ & 0 & 6 & 6 & 10 & 68 & 90 \\
\hline & & $\%$ & $0 \%$ & $7 \%$ & $7 \%$ & $11 \%$ & $76 \%$ & $100 \%$ \\
\hline & \multirow{2}{*}{ Enfermería } & $\mathrm{N}^{\circ}$ & 0 & 8 & 13 & 16 & 48 & 85 \\
\hline & & $\%$ & $0 \%$ & $9 \%$ & $15 \%$ & $19 \%$ & $56 \%$ & $100 \%$ \\
\hline & \multirow{2}{*}{ Obstetricia } & $\mathrm{N}^{\circ}$ & 1 & 3 & 17 & 18 & 46 & 85 \\
\hline & & $\%$ & $1 \%$ & $4 \%$ & $20 \%$ & $21 \%$ & $54 \%$ & $100 \%$ \\
\hline & Terapia & $\mathrm{N}^{\circ}$ & 1 & 4 & 6 & 8 & 63 & 82 \\
\hline & física & $\%$ & $1 \%$ & $5 \%$ & $7 \%$ & $10 \%$ & $77 \%$ & $100 \%$ \\
\hline \multirow[t]{2}{*}{ Total } & & $\mathrm{N}^{\circ}$ & 2 & 21 & 42 & 52 & 225 & 342 \\
\hline & & $\%$ & $1 \%$ & $6 \%$ & $12 \%$ & $15 \%$ & $66 \%$ & $100 \%$ \\
\hline Hábitos alimentarios & EAP & & Nunca & Casi nunca & A veces & Casi siempre & Siempre & Total \% \\
\hline \multirow{8}{*}{$\begin{array}{c}\text { Consumo de } \\
\text { desayuno diario }\end{array}$} & \multirow{2}{*}{ Nutrición } & $\mathrm{N}^{\circ}$ & 0 & 1 & 9 & 22 & 58 & 90 \\
\hline & & $\%$ & $0 \%$ & $1 \%$ & $10 \%$ & $24 \%$ & $64 \%$ & $100 \%$ \\
\hline & \multirow{2}{*}{ Enfermería } & $\mathrm{N}^{\circ}$ & 1 & 6 & 31 & 23 & 24 & 85 \\
\hline & & $\%$ & $1 \%$ & $7 \%$ & $36 \%$ & $27 \%$ & $28 \%$ & $100 \%$ \\
\hline & \multirow{2}{*}{ Obstetricia } & $\mathrm{N}^{\circ}$ & 2 & 1 & 27 & 24 & 31 & 85 \\
\hline & & $\%$ & $2 \%$ & $1 \%$ & $32 \%$ & $28 \%$ & $36 \%$ & $100 \%$ \\
\hline & \multirow{2}{*}{$\begin{array}{l}\text { Terapia } \\
\text { física } \\
\end{array}$} & $\mathrm{N}^{\circ}$ & 0 & 4 & 19 & 24 & 35 & 82 \\
\hline & & $\%$ & $0 \%$ & $5 \%$ & $23 \%$ & $29 \%$ & $43 \%$ & $100 \%$ \\
\hline \multirow[t]{2}{*}{ Total } & & $\mathrm{N}^{\circ}$ & 3 & 12 & 86 & 93 & 148 & 342 \\
\hline & & $\%$ & $1 \%$ & $4 \%$ & $25 \%$ & $27 \%$ & $43 \%$ & $100 \%$ \\
\hline \multirow{8}{*}{$\begin{array}{l}\text { Consumo de frutas a } \\
\text { diario }\end{array}$} & \multirow{2}{*}{ Nutrición } & $\mathrm{N}^{\circ}$ & 0 & 5 & 15 & 30 & 40 & 90 \\
\hline & & $\%$ & $0 \%$ & $6 \%$ & $17 \%$ & $33 \%$ & $44 \%$ & $100 \%$ \\
\hline & \multirow{2}{*}{ Enfermería } & $\mathrm{N}^{\circ}$ & 1 & 4 & 33 & 34 & 13 & 85 \\
\hline & & $\%$ & $1 \%$ & $6 \%$ & $39 \%$ & $40 \%$ & $15 \%$ & $100 \%$ \\
\hline & \multirow{2}{*}{ Obstetricia } & $\mathrm{N}^{\circ}$ & 0 & 8 & 41 & 30 & 6 & 85 \\
\hline & & $\%$ & $2 \%$ & $9 \%$ & $48 \%$ & $35 \%$ & $7 \%$ & $100 \%$ \\
\hline & \multirow{2}{*}{$\begin{array}{l}\text { Terapia } \\
\text { física } \\
\end{array}$} & $\mathrm{N}^{\circ}$ & 0 & 6 & 32 & 20 & 24 & 82 \\
\hline & & $\%$ & $0 \%$ & $7 \%$ & $39 \%$ & $24 \%$ & $29 \%$ & $100 \%$ \\
\hline \multirow[t]{2}{*}{ Total } & & $\mathrm{N}^{\circ}$ & 1 & 23 & 121 & 114 & 83 & 342 \\
\hline & & $\%$ & $1 \%$ & $7 \%$ & $35 \%$ & $33 \%$ & $24 \%$ & $100 \%$ \\
\hline \multirow{6}{*}{$\begin{array}{l}\text { Realización de cinco } \\
\text { momentos de comida } \\
\text { al día }\end{array}$} & \multirow{2}{*}{ Nutrición } & $\mathrm{N}^{\circ}$ & 5 & 11 & 28 & 24 & 22 & 90 \\
\hline & & $\%$ & $6 \%$ & $12 \%$ & $31 \%$ & $27 \%$ & $24 \%$ & $100 \%$ \\
\hline & \multirow{2}{*}{ Enfermería } & $\mathrm{N}^{\circ}$ & 11 & 27 & 33 & 10 & 4 & 85 \\
\hline & & $\%$ & $13 \%$ & $32 \%$ & $39 \%$ & $12 \%$ & $5 \%$ & $100 \%$ \\
\hline & \multirow{2}{*}{ Obstetricia } & $\mathrm{N}^{\circ}$ & 12 & 25 & 27 & 12 & 9 & 85 \\
\hline & & $\%$ & $14 \%$ & $29 \%$ & $32 \%$ & $14 \%$ & $11 \%$ & $100 \%$ \\
\hline
\end{tabular}




\begin{tabular}{ccccccccc}
\hline & Terapia & $\mathrm{N}^{\circ}$ & 12 & 22 & 37 & 8 & 3 & 82 \\
& física & $\%$ & $15 \%$ & $27 \%$ & $45 \%$ & $10 \%$ & $4 \%$ & $100 \%$ \\
\hline \multirow{2}{*}{ Total } & & $\mathrm{N}^{\circ}$ & 40 & 85 & 125 & 54 & 38 & 342 \\
& & $\%$ & $12 \%$ & $25 \%$ & $37 \%$ & $16 \%$ & $11 \%$ & $100 \%$ \\
\hline
\end{tabular}

Nota: $\mathrm{EAP}=$ Escuela Académica Profesional

El consumo de alimentos procesados en estudiantes universitarios es una práctica común en países de Latinoamérica [15]. El 46\% del total de estudiantes, a veces consumen productos procesados. Los estudiantes encuestados de Nutrición en un $39 \%$ casi nunca lo consumen, mientras que solo el $4 \%$ del total siempre consume productos procesados. Tal como se observa en la Tabla 3. Estos resultados son semejantes a estudios internacionales relativos al consumo de esta clase de alimentos por estudiantes universitarios [16]. Es evidente que el bajo porcentaje de estudiantes que consumen siempre productos procesados implica que poseen la información debida, es decir, el conocimiento sobre las consecuencias negativas para con la salud que se produce por el consumo constante de estos tipos de productos. Se precisa que en nuestro país hay leyes que de alguna forma promueven la adecuada alimentación correcta y la salud para la población en general como es la normativa de la Ley de promoción de la alimentación saludable para niños, niñas y adolescentes $\mathrm{N}^{\circ}$ 30021. Documento importante que promueve la buena y correcta elección de productos de consumo humano con una orientación para la elección de los mismos basado en el contenido de las etiquetas.

El proceder a la lectura concentrada de una etiqueta de un producto procesado creado para la alimentación humana puede permitir la prevención de diversas enfermedades. Así como también el observar la fecha de caducidad de dichos alimentos mejora la seguridad alimentaria y evita contraer enfermedades por intoxicación [17]. El 66\% del total de estudiantes de Ciencias de la Salud siempre toma en cuenta la fecha de vencimiento antes de consumir un producto envasado. Los estudiantes de Nutrición (76\%) y Terapia física $(77 \%)$ poseen el porcentaje más alto por carrera profesional. (Tabla 3). Últimamente, se toma especial cuidado en la salud el tener este buen hábito que previene de posibles intoxicaciones que pueden perjudicarnos. Cabe precisar que en nuestro país se evidencia factores que influyen en este proceso de darnos el tiempo necesario para observar la fecha de vencimiento y el etiquetado de los alimentos [18].

Tomar desayuno en el momento adecuado, consumir frutas $y$, en el almuerzo, ensaladas son hábitos que se aprenden en casa. El $43 \%$ del total de estudiantes de Ciencias de la Salud siempre desayuna, obteniendo porcentajes altos los estudiantes de Nutrición (64\%) y Terapia física $(43 \%)$, pero se debe precisar que un $25 \%$ del total a veces realiza este primer momento de comida. Se observa que los estudiantes de Enfermería y Obstetricia poseen los porcentajes más altos por carrera que a veces desayuna. (Tabla 3). Se precisa que son resultados aceptables ya que se fundamenta que la principal comida al iniciar el día categóricamente es el desayuno, puesto que otorga energía necesaria para rendir de la mejor manera en el estudio. Una investigación realizada a estudiantes en Chile expresa que los estudiantes universitarios, debido a su autonomía, pueden elegir qué comer y de esta manera establecen ciertos hábitos que influirán en su alimentación [19].

El $33 \%$ del total de estudiantes casi siempre consume frutas a diario. La Escuela Académica Profesional de Enfermería posee -comparando por carrera- el porcentaje de estudiantes más alto con un $40 \%$. Un $35 \%$ del total a veces consume frutas. Solo un $7 \%$ casi nunca las consume.

Si se realiza una comparación por carrera profesional, Nutrición posee un mayor porcentaje de estudiantes que siempre consumen frutas. Ello es posible debido a que no solo en la asignatura en mención se le explica los beneficios de este tipo de alimentos, sino que tiene que ver con la naturaleza de la carrera. (Tabla 3). Es beneficioso el consumo de frutas en la media mañana y media tarde. El consumo de frutas, evidentemente debido a sus cualidades por el contenido de agua, fibra y vitaminas se hace necesario y primordial para toda persona [20]. Los resultados, respecto al consumo de frutas se asemeja a una investigación realizada a estudiantes en Colombia que considera relevante 
el consumo de este alimento como una manera de prevenir enfermedades [21].

El $11 \%$ del total de estudiantes de Ciencias de la Salud tiene siempre cinco momentos de comida al día y casi siempre un 16\%. Los estudiantes de Nutrición poseen el porcentaje más alto por carrera considerando los que siempre realizan estos cinco momentos de comida. Por el contrario, los estudiantes de Enfermería casi nunca realizan estos cinco momentos (32\%). Cabe resaltar que un $37 \%$ del total a veces cumplen esos cinco momentos de comida. Destacan los estudiantes de Enfermería y Terapia física con valores porcentuales altos por carrera. (Tabla 3). En un estudio realizado en tres universidades de Bogotá se expresa que la elección de las comidas se toma en consideración el factor tiempo y no obvian el desayuno y cena que consideran momentos importantes de alimentación [22]. Se debe señalar que debido a múltiples actividades que se dan en las aulas universitarias, el tiempo que se toma para el desarrollo de las asignaturas y la distancia del lugar de residencia son algunos factores que repercute en el no cumplimiento de este hábito. Se considera además relevante el consumo alimenticio para el progreso de una colectividad [23].

Sobre la forma cómo las personas realizan el proceso de distribución de comidas, al realizarlas de manera regular en un horario en la que se llega a tener más de tres momentos de comida diaria puede provocar una mejora en el ámbito laboral y estudiantil. Claro está que debe prevalecer una cantidad de nutrientes suficiente en esos momentos de comida para un efecto positivo en el organismo considerando las medidas antropométricas de talla y el peso de los estudiantes. [24]. Por lo tanto, el horario de estudios 0 el tráfico generalizado actualmente en la ciudad de Lima a diversas horas del día, repercute en la no realización de esos momentos de comida necesarios. Así como también los hábitos de las familias de los estudiantes en mención.

\section{Conclusiones}

Es evidente que la información y educación nutricional es necesaria y positiva así como también ello se vea aunado a una práctica alimentaria adecuada. Sobre todo en personas que tienen formación profesional relativa a la salud por el que debe haber un mayor compromiso, puesto que en el campo laboral se trata con otros seres humanos a las que se les debe orientar sobre la relevancia de unos hábitos adecuados de alimentación.
Los estudiantes de la universidad materia del estudio sí poseen de manera general una educación nutricional y hábitos alimentarios moderados, aceptables, mas no sobresalientes. Los estudiantes de Nutrición y dietética destacan sobre las demás carreras de Ciencias de la Salud en resultados porcentuales de Educación nutricional y Hábitos alimentarios. No se encontró una gran diferencia entre las carreras mencionadas respecto a la educación nutricional recibida y los hábitos alimentarios.

Si bien es cierto la educación nutricional recibida puede -de cierta forma- influir en los hábitos alimentarios es necesario que se produzca el abandono de hábitos inadecuados para de esta manera tener y formar una cultura alimenticia ideal y de esta forma repercutir positivamente cuando dichos profesionales ingresen al campo laboral respectivo. Se podría realizar más adelante un estudio de una mayor amplitud considerando a todos los profesionales de diversas carreras de Ciencias de la salud de otras universidades privadas que con mayor motivo deben tener unos hábitos y educación nutricional sobresaliente y a la altura de las necesidades que la población en general requiere y solicita.

\section{Agradecimientos}

A la Universidad Privada del Norte, en especial a la Coordinación del Área de Nutrición por darnos las facilidades para la aplicación de los instrumentos. A los docentes de los diversos turnos y carreras, por su disposición que permitieron aplicarlas y a los estudiantes que nos brindaron su tiempo y cooperación.

\section{Referencias}

[1] M.A. Sánchez, E. García y M.S. Naupari, Educación alimentaria y nutricional. (Universidad Nacional de Educación Enrique Guzmán y Valle, La Cantuta, 2014).

[2] A.M. Castiblanco, MSc. Tesis, Universidad Peruana Unión, 2017.

[3] F. Huisacayna, L. Ninahuaman y $M$. Figueroa, Revista Enfermería Vanguardia, 5 (2017) 77-86.

[4] C. Torres, C. Trujillo, A.L. Urquiza, R, Salazar y A. Taype, Revista Chilena de Nutrición, 43 (2016)146-154.

[5] G. Aguilar, MSc. Tesis, Universidad Andina Néstor Cáceres Velásquez, 2015. 
[6] L. Palomares, MSc. Tesis, Universidad Peruana de Ciencias Aplicadas, 2014.

[7] L. Serra-Majem, L. Ribas, A, García, C. Pérez-Rodrigo y J. Aranceta, Eur J Clin Nutr. 57(2003)9.

[8] L. Puerto y P. Tejero, Nutrición Clínica y Dietética Hospitalaria, 33(2013) 56-65.

[9] S. Palenzuela, A. Perez, L. Pérula, J. Fernandez y J. Maldonado, Anales del sistema sanitario de Navarra, 37(2014) 4758.

[10] J. Pereira y M. Salas, Revista Electrónica Educare, 21(2017) 1-23.

[11] M. Serrano y J. Mateos, Nutrición y alimentación: Nuevas perspectivas (Mc Graw Hill, España, 2008) pp. 122.

[12] F. Hernández, C. Fernández y P. Baptista. Metodología de la Investigación. (Mc Graw Hill, México, 2014), pp. 613.

[13] C. Iglesias, et al., Nutrición Hospitalaria, 26(2011) 27-36.

[14] C. García, J. Carreón, J. Hernández, M.M. López y J.M. Bustos. Revista Latinoamericana Polis. 12(2013) 364-366.

[15] A. De Piero, N. Bassett, A. Rossi y N. Sammán. Nutrición Hospitalaria, 31(2015) 1824-1831.
[16] F. Becerra, y M. Vargas. Revista Salud Pública, 17(2015) 778-770.

[17] M. L. Lázaro and C. H. Domínguez, Guía alimentaria para la población peruana. Editado por El Ministerio de Salud. (Minsa, Lima, 2019) pp. 60

[18] A. P. Cornejo y L. C. Liza, MSc. Tesis. Universidad Peruana de Ciencias Aplicadas, 2015.

[19] S. Duran, Revista Médica de Chile, 145 (2017) 1403-1411.

[20] M. Rodríguez, Revista de la Facultad de Medicina Humana, 19(2019) $105-111$.

[21] L. Restrepo, L. Urango y G. Deossa, Revista Chilena de Nutrición, 41(2014) 236-242.

[22] C. Duarte, D. Ramos, A. Latorre y P. González, Revista Salud Pública 17(2015) 925-937.

[23] C. Martínez y A. Rodríguez, Offarm 21(2002) 80-86.

[24] A. Laguna, Hábitos alimenticios, peso y metabolismo. (Universidad Autónoma de México, México, 2015) p. 37 